University of Nebraska - Lincoln

DigitalCommons@University of Nebraska - Lincoln

Faculty Publications, Department of Physics and Astronomy

$11-17-2005$

\title{
Nanomesa and nanowell formation in Langmuir-Blodgett polyvinylidene fluoride trifluoroethylene copolymer films
}

\author{
Jiangyu Li \\ University of Nebraska-Lincoln, jjli@uw.edu \\ Yang Luo \\ University of Nebraska-Lincoln \\ Mengjun Bai \\ University of Missouri-Columbia, baime@missouri.edu \\ Stephen Ducharme \\ University of Nebraska-Lincoln, sducharme1@unl.edu
}

Follow this and additional works at: https://digitalcommons.unl.edu/physicsfacpub

Part of the Physics Commons

Li, Jiangyu; Luo, Yang; Bai, Mengjun; and Ducharme, Stephen, "Nanomesa and nanowell formation in Langmuir-Blodgett polyvinylidene fluoride trifluoroethylene copolymer films" (2005). Faculty Publications, Department of Physics and Astronomy. 10.

https://digitalcommons.unl.edu/physicsfacpub/10

This Article is brought to you for free and open access by the Research Papers in Physics and Astronomy at DigitalCommons@University of Nebraska - Lincoln. It has been accepted for inclusion in Faculty Publications, Department of Physics and Astronomy by an authorized administrator of DigitalCommons@University of Nebraska Lincoln. 


\title{
Nanomesa and nanowell formation in Langmuir-Blodgett polyvinylidene fluoride trifluoroethylene copolymer films
}

\author{
Jiangyu $\mathrm{Li}^{\mathrm{a})}$ and Yang Luo \\ Department of Engineering Mechanics and Center for Materials Research and Analysis, \\ University of Nebraska-Lincoln, Lincoln, Nebraska 68588-0526 \\ Mengjun Bai ${ }^{\text {b) }}$ and Stephen Ducharme \\ Department of Physics and Astronomy and Center for Materials Research and Analysis, \\ University of Nebraska-Lincoln, Lincoln, Nebraska 68588-0111
}

(Received 27 June 2005; accepted 27 September 2005; published online 17 November 2005)

\begin{abstract}
In this letter, we report an energetics-based model to explain the self-organizing nanomesa and nanowell patterns recently observed in Langmuir-Blodgett (LB) films of polyvinylidene fluoride trifluoroethylene $[\mathrm{P}(\mathrm{VDF}-\mathrm{TrFE})]$ copolymers. The feature size of nanomesas and nanowells has been estimated using a linear stability analysis, and the morphology of the nanomesas and nanowells has been revealed by the numerical simulation, both in good agreement with experiments. A number of other model predictions regarding the nanomesa and nanowell formation also agree with experimental observations. The model can be used to guide the design and optimization of nanostructures in the P(VDF-TrFE) LB films and possibly in other thin polymer films. (C) 2005 American Institute of Physics. [DOI: 10.1063/1.2136220]
\end{abstract}

Self-organizing and self-patterning can occur in materials over many length scales by competing atomic or molecular interactions, ${ }^{1}$ and have been observed in many different material systems. ${ }^{2-6}$ They are not only appealing scientifically, by revealing the intrinsic atomic and molecular interactions that might be difficult to detect otherwise, but may also hold the key for the development of novel materials and structures with a variety of feature sizes and enhanced functionality. ${ }^{7-10}$ As such, it is of both fundamental and technological importance to understand self-organizing at various length scales.

A remarkable crystalline nanomesa and nanowell formation has recently been discovered in Langmuir-Blodgett (LB) films of polyvinylidene fluoride trifluoroethylene $[\mathrm{P}(\mathrm{VDF}-\mathrm{TrFE})]$ copolymers after annealing. ${ }^{11}$ The atomic force microscopy (AFM) image of a 1-ML annealed film (see Fig. 1) shows an array of predominately disc-shaped nanomesas isolated from each other on the substrate, with a diameter of $95 \pm 22 \mathrm{~nm}$ and thickness of around $8.7 \pm 0.4 \mathrm{~nm}$. As the monolayers increase, more nanomesas developsome joined into extended shapes, and tending toward percolation as the number of nanomesas increases. The 4-ML films are mostly filled in, resulting in a complementary pattern of nanowells with a diameter of $128 \pm 37 \mathrm{~nm}$ and thickness of $9.8 \pm 3.3 \mathrm{~nm}$. At $8 \mathrm{ML}$ and thicker, the films remain continuous without nanowells, even after lengthy annealing. Combined with the ferroelectricity and piezoelectricity of $\mathrm{P}(\mathrm{VDF}-\mathrm{TrFE}) \mathrm{LB}$ films, ${ }^{11,12}$ the nanomesas are attractive for a number of applications including high-density nonvolatile random-access memories, acoustic transducer arrays, and infrared imaging arrays. They may also provide useful templates for the self-assembly of novel ferroelectric nanostructures. For their technological potential to be fully realized,

\footnotetext{
${ }^{\text {a) }}$ Author to whom correspondence should be addressed; electronic mail: jli2@unl.edu

${ }^{b}$ Present address: Department of Physics and Astronomy, University of Missouri-Columbia, Columbia, MI 65217.
}

however, it is essential to understand the mechanism and process of nanomesa and nanowell formation, which will not only shed light on the complicated molecular interactions in $\mathrm{P}$ (VDF-TrFE) LB films, but will also enable the the design and optimization of novel materials, structures, and devices. This is the intent of this letter.

We propose that the formation of nanomesas and nanowells reduces the free energy of the system consisting of the film and substrate. The contributions to the energy include a surface term residing in $\mathrm{P}(\mathrm{VDF}-\mathrm{TrFE})$ film and a bulk term residing in the film and substrate

$$
G=\int \Pi d A+\int W d V,
$$

where $\Pi$ is the surface energy density and $W$ is the elastic energy density. We emphasize that the surface energy density incorporates all relevant molecular interactions in $\mathrm{P}(\mathrm{VDF}-$ TrFE), and takes the following form:

$$
\Pi=\alpha C_{, \beta} C_{, \beta}+g(C)+\gamma_{\alpha \beta}(C) \varepsilon_{\alpha \beta},
$$

where the first term penalizes the gradient of surface concentration $C$ of $\mathrm{P}(\mathrm{VDF}-\mathrm{TrFE})$ monomer, and thus leads to interface energy between different patterns. Greek subscripts range from 1 to 2 and repeated Greek subscripts are summed from 1 to 2 ; the subscript comma is used to denote differentiation. The second term is the internal energy density per unit surface area, which we assume is a function of film thickness $h$. Microscopically, this assumption implies that the internal energy density of $\mathrm{P}(\mathrm{VDF}-\mathrm{TrFE})$ depends on its morphology. Indeed, it is well known that lamellar polymer crystals possess preferred thickness due to chain folding, ${ }^{13-15}$ which was shown to be thermodynamically stable by Monte Carlo simulation. ${ }^{16}$ A similar assumption has also been made for thin nematic films experiencing spinodal dewetting, ${ }^{17}$ and we expect it will hold for LB films with thickness in the nanometer range. In particular, we assume that $g(C)=a h_{r}^{2}\left[C / P h_{r}-1\right]^{2} C^{2}$, which possesses two 

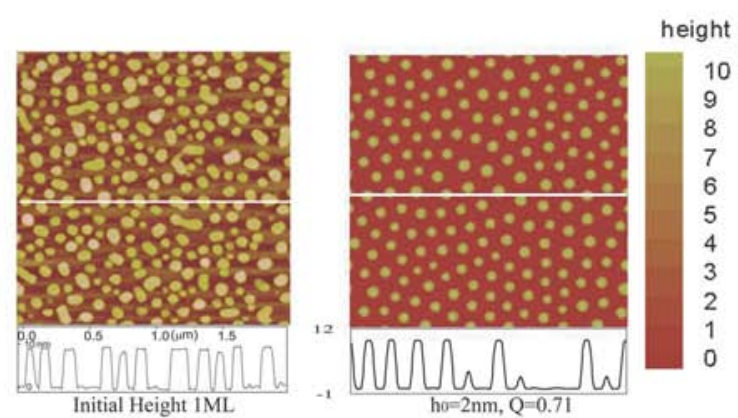

(a)
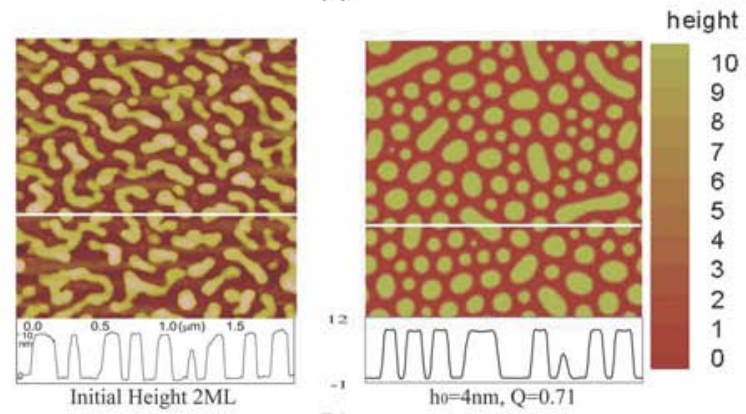

(b)
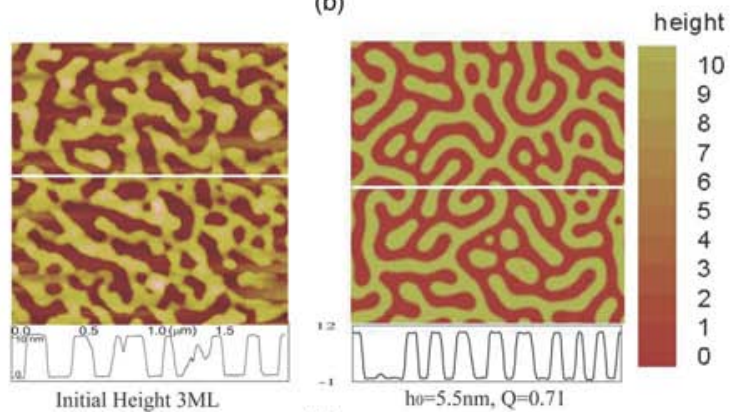

(c)
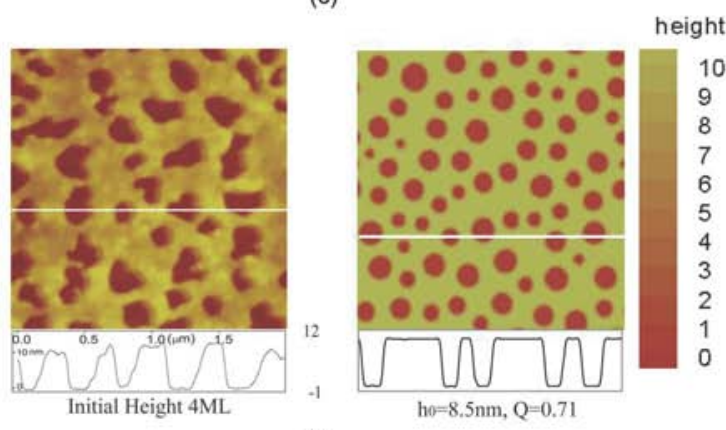

(d)

FIG. 1. Images of nanomesas and nanowells and the corresponding height profiles recorded along the white lines in the images. (Left) Atomic force microscopy measurements; (right) numerical simulations.

minima at thicknesses of 0 and $h_{r}$, with $P=C / h$ being the volume concentration of $\mathrm{P}(\mathrm{VDF}-\mathrm{TrFE})$ that is conserved during the pattern evolution. The third term in (2) is a surface energy due to the surface stress $\gamma_{\alpha \beta}$ and strain $\varepsilon_{\alpha \beta}$, and we assume surface stress varies with surface concentration linearly, ${ }^{18} \gamma_{\alpha \beta}(C)=\gamma \delta_{\alpha \beta} C$, where $\delta_{\alpha \beta}$ is the Kronecker delta. From elastic equilibrium, it is established that the stress $\sigma$ on the surface satisfies $\sigma_{3 \alpha}=\gamma_{\alpha \beta, \beta}$ and $\sigma_{33}=0$. As a result, the gradient of surface stress serves as shear traction on the film surface, leading to a stress field and elastic energy within the film and substrate. By combining the elastic energy in the film and substrate, and surface stress energy in the film, we obtain $^{3}$

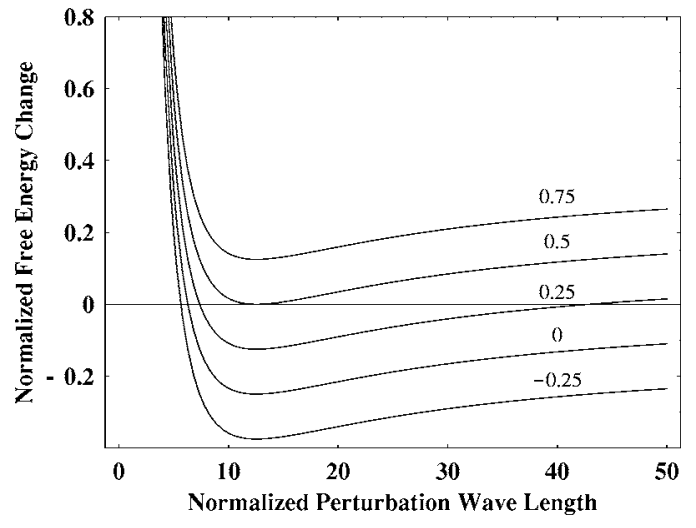

FIG. 2. The change of normalized free energy $\Delta G$ as a function of the perturbation wavelength $\lambda / l$ at different $\mu$.

$$
\int W d V+\int \gamma_{\alpha \beta} \varepsilon_{\alpha \beta} d A=-\frac{1}{2} \int \gamma_{\alpha \beta, \beta} u_{\alpha} d A,
$$

which prefers a nonuniform distribution of surface concentration; in (3), $u_{\alpha}$ is the displacement of the film.

It is clear that we have three different interactions competing with each other in $\mathrm{P}(\mathrm{VDF}-\mathrm{TrFE})$ films. In particular, the internal energy density per unit surface area possesses two minima, and thus will result in pattern separation. The interface energy will drive the pattern coarsening to minimize the interface area, while the elasticity will drive the pattern refining to minimize the elastic energy. The interplay between coarsening and refining determines the feature size of nanomesas and nanowells. From the variation of free energy, the driving force for the pattern evolution is obtained as

$$
F_{\alpha}=-\left(\frac{\partial g}{\partial C}-2 \alpha \nabla^{2} C+\gamma \varepsilon_{\beta \beta}\right),{ }_{\alpha},
$$

where $\nabla^{2}=\partial^{2} / x_{1}^{2}+\partial^{2} / x_{2}^{2}$, leading to the evolution equation of the concentration field

$$
\frac{\partial C}{\partial t}=M \nabla^{2}\left(\frac{\partial g}{\partial C}-2 \alpha \nabla^{2} C+\gamma \varepsilon_{\alpha \alpha}\right),
$$

where $M$ is molecular mobility. The strain field is determined by solving an elastic problem, ${ }^{3}$

$$
\varepsilon_{\alpha \alpha}=\frac{\left(\nu^{2}-1\right) \gamma}{\pi E} \iint \frac{\left(x_{1}-\xi_{1}\right) \frac{\partial C}{\partial \xi_{1}}+\left(x_{2}-\xi_{2}\right) \frac{\partial C}{\partial \xi_{2}}}{\left[\left(x_{1}-\xi_{1}\right)^{2}+\left(x_{2}-\xi_{2}\right)^{2}\right]^{3 / 2}} d \xi_{1} d \xi_{2},
$$

where $E$ and $\nu$ are the Young's modulus and Poisson's ratio of the film-substrate composite system. Since the substrate is much stiffer than polymer film, most of the deformation will occur in polymer.

The competition between the concentration gradient coarsening and elastic deformation refining leads to a length defined by $l=E \alpha /\left(1-\nu^{2}\right) \gamma^{2}$, which scales with the feature size of nanomesas and nanowells. To see this, we consider a small perturbation on a uniform surface concentration field $C\left(x_{1}\right)=C_{0}+A \sin k x_{1}$, where $A$ and $k=2 \pi / \lambda$ are the amplitude and the wave number of the dominant perturbation. Expanding $g(C)$ around $C_{0}$ and comparing the free energy before and after perturbation, we obtain $\Delta G /\left(\alpha A^{2} / 2 l^{2}\right)$ $=\mu / 2-2 \pi l / \lambda+(2 \pi l / \lambda)^{2}$, where $\mu=\alpha g_{2}\left(E / 1-\nu^{2}\right)^{2} / \gamma^{4}$ and 
$g_{2}=2 a h_{r}^{2}\left[6\left(h_{0} / h_{r}\right)^{2}-6 h_{0} / h_{r}+1\right]$. It is clear that $\Delta G=0$ has one solution for $\lambda / l$ at $\mu=0.5$, no solution at $\mu>0.5$, and two solutions at $\mu<0.5$, as shown in Fig. 2. This suggests that the free energy of the system cannot be reduced by surface concentration perturbation when $\mu>0.5$, so that the uniform surface concentration is stable. When $\mu<0.5$, the free energy can be reduced by a surface concentration perturbation of a certain wavelength, indicating that the uniform surface concentration is unstable. The free energy is then minimized by $\lambda=4 \pi l$, suggesting an equilibrium feature size for nanomesas or nanowells. A similar change of stability has been observed in binary epilayers. ${ }^{19}$

Our model offers a number of predictions on the nanomesa and nanowell patterns that are consistent with experiments. ${ }^{11}$ First, we predict that the feature size scales with $4 \pi l$, which is proportional to the elastic constant $E /$ $\left(1-\nu^{2}\right)$ of the film and inversely proportional to $\gamma^{2}$, with $\gamma$ being the surface stress coefficient of the film. This is confirmed by experimental observation that nanomesa size on an aluminum-coated silicon substrate is smaller than that on a noncoated silicon substrate. ${ }^{20}$ Aluminum does have smaller stiffness than silicon, although interface interactions and substrate roughness may also play a role. As the temperature increases, it is reasonable to assume that the surface stress in the film will be partially relieved, resulting in a larger nanomesa size; this is also consistent with experimental observation. In addition, the feature size is independent of the initial thickness of the film in our model, while in experiments, it is observed that with the increase of the film's initial thickness, the smallest length scale in the nanomesa and nanowell patterns remains roughly the same. Second, we predict that there is a critical $\mu=0.5$, above which the uniform surface concentration is stable against perturbation. This suggests that there is a critical initial thickness, above which the nanomesa or nanowell will not form. Indeed, no nanomesas or nanowells are observed in $\mathrm{P}(\mathrm{VDF}-\mathrm{TrFE})$ films of $8 \mathrm{ML}$ and thicker. We also notice that $\mu$ is proportional to $\alpha$, the coefficient for interface energy, indicating that large interface energy will prevent the formation of nanomesa and nanowells. This is also supported by the experimental observation that nanomesas and nanowells only form in the paraelectric phase and are not observed in the ferroelectric phase. While the mobility of the ferroelectric phase may play a role here, it may also be due to larger interface energy in the ferroelectric phase caused by the depolarization field.

Finally, we have implemented the model using a finite difference method and fast Fourier transform technique, with the objective to enable the simulation guided design and optimization of nanostructures in the P(VDF-TrFE) LB films and possibly other thin polymer films. Typical results are shown in Fig. 1, which are consistent with experiments. The details of the numerical simulations will be reported elsewhere.

The authors acknowledge the support of the National Science Foundation Nanomanufacturing Program (DMI0300014) and the American Chemical Society Petroleum Research Fund Type G Grant (PRF No. 39526-G5B).

\footnotetext{
${ }^{1}$ M. Muthukumar, C. K. Ober, and E. L. Thomas, Science 277, 1225 (1997).

${ }^{2}$ M. Seul and V. S. Chen, Phys. Rev. Lett. 70, 1658 (1993).

${ }^{3}$ Z. Suo and W. Lu, J. Mech. Phys. Solids 48, 211 (2000).

${ }^{4}$ L. Proville, Phys. Rev. Lett. 88, 046102 (2002).

${ }^{5}$ B. Yang, F. Liu, and M. G. Lagally, Phys. Rev. Lett. 92, 025502 (2004).

${ }^{6}$ Z. Lin and S. Granick, J. Am. Chem. Soc. 127, 2816 (2005).

${ }^{7}$ Q. M. Zhang, H. F. Li, M. Poh, F. Xia, Z. Y. Cheng, H. S. Xu, and C. Huang, Nature (London) 419, 284 (2002).

${ }^{8}$ J. Y. Li, Phys. Rev. Lett. 90, 217601 (2003).

${ }^{9}$ Z. M. Dang, Y. Shen, and C. W. Nan, Appl. Phys. Lett. 81, 4814 (2002).

${ }^{10}$ H. Zeng, J. Li, J. P. Liu, Z. L. Wang, and S. H. Sun, Nature (London) 420, 395 (2002).

${ }^{11}$ M. J. Bai and S. Ducharme, Appl. Phys. Lett. 85, 3528 (2004).

${ }^{12}$ A. V. Bune, V. M. Fridkin, S. Ducharme, L. M. Blinov, S. P. Palto, A. V. Sorokin, S. G. Yudin, and A. Zlatkin, Nature (London) 391, 874 (1998).

${ }^{13}$ D. M. Sadler, Nature (London) 326, 174 (1987).

${ }^{14}$ T. Yamamoto, J. Chem. Phys. 107, 2653 (1997).

${ }^{15}$ P. Welch and M. Muthukumar, Phys. Rev. Lett. 87, 218302 (2001).

${ }^{16}$ J. P. K. Doye and D. Frenkel, Phys. Rev. Lett. 81, 2160 (1998).

${ }^{17}$ F. Vandenbrouck, M. P. Valignat, and A. M. Cazabat, Phys. Rev. Lett. 82, 2693 (1999).

${ }^{18}$ A. Grossmann, W. Erley, and H. Ibach, Surf. Sci. 337, 183 (1995).

${ }^{19}$ W. Lu and Z. Suo, Z. Metallkd. 90, 956 (1999).

${ }^{20}$ M. J. Bai and S. Ducharme (submitted).
} 